\title{
Pacific
}

Journal of

Mathematics

\section{ON THE MINIMAL FREE RESOLUTION OF GENERAL} EMBEDDINGS OF CURVES

EDOARDO BALLICO 


\title{
ON THE MINIMAL FREE RESOLUTION OF GENERAL EMBEDDINGS OF CURVES
}

\author{
EdoARDo BALLICO
}

\begin{abstract}
Here we study the minimal free resolution of general embeddings in $\mathbf{P}^{n}$ of genus $g$ curves with general moduli. We prove that if $p$ is an integer with, roughly, $g \leq n^{2} /(2 p+2)$, then the embedding has the property $N_{p}$, i.e., the first $p$ pieces of the resolution are as simple as possible.
\end{abstract}

We work over an algebraically closed field. Let $C$ be a smooth curve embedded in $\mathbf{P}^{n}$. We are interested in the minimal free resolution of $C$. Here we will consider the case in which the curve has general moduli and the embedding is general. Recall the following definition $([\mathbf{5}],[\mathbf{6}])$.

Definition 0.1. Let $C \subset \mathbf{P}^{n}$ be a reduced curve; fix an integer $p \geq 1$; $C$ satisfies the property $N_{p}$ if $C$ is arithmetically Cohen - Macaulay and for every integer $i$ with $1 \leq i \leq p$ the $i^{\text {th }}$-sheaf appearing in the minimal free resolution of the homogeneus ideal of $C$ is the direct sum of line bundles of degree $-i-1$.

For instance if we say that $N_{0}$ means " $C$ is arithmetically Cohen-Macaulay", then $N_{1}$ means that the curve $C$ is $N_{0}$ and its homogeneous ideal is generated by quadrics. Furthermore, if $p>0$, then $N_{p}$ implies $N_{p-1}$.

In this paper, using degeneration techniques, we will prove the following results (Theorems 0.2 and 0.3 ).

Theorem 0.2. Fix an integer $p \geq 1$. For every integer $u$, set:

$$
\alpha_{p}(u):=\left(u^{2}\right) /(2 p+2)-(u / 2)
$$

Fix an integer $n \geq 3$ with $n \geq p+1$, and set:

$$
G_{p}(n):=\alpha_{p}((p+1)[n /(p+1)])
$$

where $[y]$ is the greatest integer $\leq y$. Then for every integer $g \leq G_{p}(n)$ the general linearly normal non special curve $C \subset \mathbf{P}^{n}$ with $p_{a}(C)=g$ and $\operatorname{deg}(C)=g+n$ satisfies the property $N_{p}$.

Note that $G_{p}(n)$ has order $\left(n^{2}\right) /(2 p+2)$ and hence $d:=g+n$ is usually much smaller than $2 g+p$ if $n$ is much larger than $p$. 
In the case of special linearly normal embeddings we have the following "conditional" result.

Theorem 0.3. Fix an integer $p \geq 1$ and an integer $s>2 p$. Assume that a general canonical curve of genus $s+1$ in $\mathbf{P}^{s}$ has the property $N_{p}$. Fix an integer $n>p+s$; write $n=s+a(p+1)+b$ with $a, b$ integers and $0 \leq b \leq p$. Set:

$$
S_{p, s}(n):=(a+1) s+1+a(a-1)(p+1) / 2 .
$$

Then for every integer $g$ with $s+1 \leq g \leq S_{p, s}(n)$ a general linearly normal curve $C \subset \mathbf{P}^{n}$ with $p_{a}(C)=g$ and $h^{1}\left(C, \mathbf{O}_{C}(1)\right)=1$ (hence of degree $g+n-1)$ has the property $N_{p}$.

Quoting existing references on $N_{p}$ for the canonical model of a curve, $C$, with general moduli (e.g. [7], [3]), one obtains corresponding statements for special embeddings of $C$. We stress that the proof of Theorems 0.2 and 0.3 , being a kind of induction on $n$ using as inductive tool Lemma 1.2, may be used to obtain many other cases not covered by the statements of 0.2 and 0.3 ; the proof of 0.2 should be helpful to the reader interested in other cases.

I want to thank the referee for several suggestions which improved very much the readability of the paper;

The author was partially supported by MURST and GNSAGA of CNR (Italy).

1. In this section we will prove Theorems 0.2 and 0.3 . A key quotation for the proofs here is the criterion for condition $N_{p}$ given in [6, Prop. 1.3.3]; the base field for all [6] was the complex number field, but the statement of the quoted criterion 1.3.3 works in arbitrary characteristic because each of the steps of its proof either works verbatim in positive characteristic or it is known to hold in general; furthermore, [6, Prop. 1.3.3], although stated only for smooth curves, works with the same proof for all reduced curves. It is the use of this criterion for $N_{p}$ which gives the condition of linear normality in the statements of $0.2,0.3$ and 1.3 .

Fix an integer $n \geq 3$; following the notations of [6], $M_{n}$ will denote the rank $n$ vector bundle on $\mathbf{P}^{n}$ with $M_{n}(-1)$ isomorphic to the cotangent bundle.

The following result is well known (see e.g. [1, Lemma 1.3]):

Lemma 1.1. Let $D \subset \mathbf{P}^{n}$ be a rational normal curve. Then $M \mid D$ is the direct sum of $n$ line bundles of degree -1 .

Lemma 1.2. Fix integers $n, p, t, j$ and $m$ with $n \geq t \geq j>p>1$ and $m \geq 0$. Let $C \subset \mathbf{P}^{n-j}$ be a reduced curve satisfying condition $N_{p}$. See $\mathbf{P}^{n-j}$ 
as a linear subspace $V$ of $\mathbf{P}^{n}$ and let $D$ be a smooth rational curve of degree $t$ in $\mathbf{P}^{n}, D$ spanning a linear subspace $W$ of dimension $t$, with $\operatorname{dim}(V \cap W)=$ $t-j$ and $\operatorname{card}(C \cap D)=t-j+1, D$ intersecting quasi transversally $C$, (hence $C \cup D$ spanning $\left.\mathbf{P}^{n}\right)$; assume that $H^{1}\left(C,\left(\Lambda^{(p+1)} M_{n-j}(1)\right)(m) \mid C\right)=0$; then $H^{1}\left(C \cup D,\left(\Lambda^{(p+1)} M_{n}(1)\right)(m) \mid(C \cup D)\right)=0$.

Proof. Consider the following Mayer-Vietoris exact sequence:

$$
\begin{aligned}
0 & \rightarrow\left(\Lambda^{(p+1)} M_{n}(1)\right)(m) \mid(C \cup D) \\
& \rightarrow\left(\Lambda^{(p+1)} M_{n}(1)\right)(m)\left|C \oplus\left(\Lambda^{(p+1)} M_{n}(1)\right)(m)\right| D \\
& \rightarrow\left(\Lambda^{(p+1)} M_{n}(1)\right)(m) \mid(C \cap D) \rightarrow 0 .
\end{aligned}
$$

Note that $M_{n} \mid C \cong\left(M_{n-j} \mid C\right) \oplus \mathbf{O}_{C}^{j}$. Hence $\Lambda^{(p+1)} M_{n} \mid C$ is a direct sum of trivial factors and factors isomorphic to $\Lambda^{u} M_{n-j} \mid C$ for some integer $u \leq p$. Since $N_{p}$ implies $N_{u}$ for every $u<p$ we have

$$
H^{1}\left(C,\left(\Lambda^{(p+1)} M_{n}(1)\right)(m) \mid C\right)=0 .
$$

Note that $M_{n} \mid D$ is the direct sum of $t$ line bundles of degree -1 and $n-t$ copies of $\mathbf{O}_{D}$. Hence $\left(\Lambda^{(p+1)} M_{n}(1)\right)(m) \mid D$ is a direct sum of line bundles of degree at least $m t+t-p-1$. To conclude it is sufficient to use (4) and to check (see below) the surjectivity of the restriction map $p: H^{0}\left(\left(\Lambda^{(p+1)} M_{n}(1)\right)(m) \mid D\right) \rightarrow H^{0}\left(\left(\Lambda^{(p+1)} M_{n}(1)\right)(m) \mid(C \cap D)\right)$. For the surjectivity of $p$, note that, since $\operatorname{deg}\left(\mathbf{O}_{D}(-(C \cap D))\right)=-\operatorname{card}(C \cap D)$ and $j>p$, we have $H^{1}(D, E(-(C \cap D)))=0$ for every line bundle $E$ on $D$ with $\operatorname{deg}(E) \geq t-p-1$.

Note that $p_{a}(C \cup D)=p_{a}(C)+t-j$ and that $C \cup D$ spans $\mathbf{P}^{n}$. An easy Mayer-Vietoris exact sequence gives $h^{1}\left(C \cup D, \mathbf{O}_{C \cup D}(1)\right)=h^{1}\left(C, \mathbf{O}_{C}(1)\right)$ and $h^{0}\left(C \cup D, \mathbf{O}_{C \cup D}(1)\right)=n+1$. Hence any smoothing of $C \cup D$ will give linearly normal smooth curves "near" $C \cup D$.

Proof of 0.2 . We fix the integer $p$. First we will prove $N_{p}$ for the genus $G_{p}(n)$ and every integer $n$. Set $n=a(p+1)+r$. As a starting point we assume the property $N_{p}$ for the rational normal curve of $\mathbf{P}^{p+1}$. Of course, better results and other example can be obtained using other curves in $\mathbf{P}^{p+1}$ with property $N_{p}$, e.g. the ones given by an important theorem of M. Green (proven in any characteristic in [5, Prop. 3.2]) saying that a linearly normal embedding of degree at least $2 k+1+p$ of any smooth curve of genus $k$ has the property. $N_{p}$. The main property of the function $G_{p}$ is the property $G_{p}(m+p+1)=$ $G_{p}(m)+(p+1)[m /(p+1)]$; its normalization $G_{p}(p+1)=0$ comes from the choice we made for the starting point of the induction. Then we apply $(a-1)$ times Lemma 1.2, always with $j=p+1$ and at each step with the maximal 
possible $t$; in the $k^{\text {th }}$-step we pass from a curve of genus $G_{p}((k+1)(p+1))$ in $\mathbf{P}^{(k+1)(p+1)}$ to a curve of arithmetic genus $G_{p}((k+2)(p+1))$ in $\mathbf{P}^{(k+2)(p+1)}$. Then we apply Lemma 1.2 for the integers $n, t, j$ with $t=n$ and $j=r$, concluding the case $g=G_{p}(n)$. Now we will check $N_{p}$ in $\mathbf{P}^{n}$ for any non negative integer $g<G_{p}(n)$ There is an integer $x \leq n-p-1, x$ divisible by $p+1$, with $G_{p}(x) \leq g<G_{p}(x+1)$, say $x=m(p+1)$. We take a curve, $C$, in $\mathbf{P}^{X}$ with $N_{p}$ and genus $G_{p}(x)$ and we apply Lemma 1.2 for the integers $n, t, j$, with $j=n-x$ and $t-j=g-G_{p}(x)$.

At each step of the induction the possibility of deforming the reducible curve to a smooth linearly normal curve (i.e., a smooth curve, $T$, with the correct $h^{1}\left(T, \mathbf{O}_{T}(1)\right)$ ) is proven (in a much stronger form than needed here and for 0.3 ) independently in several papers: see for instance [BE, Lemma $1.2,(1)]$, or $[4$, Th.4.1], or $[8, \S 5]$; one can also see a discussion of the way the smoothing concerns the moduli spaces in $[8]$ and $[2, \S 1, \S 2, \S 3]$.

Note that the bound (2) on the genus is just a byproduct of the inductive proof.

Proof of 0.3 . The proof of 0.2 works with the following modifications. Instead of quoting [6, Prop. 1.3.3], use Remark (2) after the proof of [6, Prop. 1.3.3]. The starting point of the induction is a general canonical curve in $\mathbf{P}^{s}$ which in the statement of 0.3 is assumed to have property $N_{p}$. To check the smoothability of reduced curves, use for instance [2, Lemma 1.2 (1)]. To check the condition " $h^{1}\left(T, \mathbf{O}_{T}(1)\right)=1$ ", first (as remarked before the proof of 0.2) use a Maver - Vietoris exact sequence to prove it for the reducible curves; then use semicontinuity to obtain that $h^{1}\left(T, \mathbf{O}_{T}(1)\right) \leq 1$ if $T$ is general, while $h^{1}\left(T, \mathbf{O}_{T}(1)\right) \geq 1$ by Riemann - Roch.

\section{References}

[1] E. Ballico, Generators for the homogeneous ideal of s general points in $\mathbf{P}^{3}$, J. Algebra, 106 (1987), 46-52.

[2] E. Ballico and Ph. Ellia, On the existence of curves with maximal rank in $\mathbf{P}^{n}$, J. reine angew. Math., 397 (1989), 1-22.

[3] D. Bayer and D. Eisenbud, Graph curves, Advances in Math., 86 (1991), 1-40.

[4] R. Hartshorne and A. Hirschowitz, Smoothing algebraic space curves, in: Algebraic Geometry - Sitgers (1983), pp. 98-131, Lect. Notes in Math., 1124 Springer-Verlag, Berlin (1985).

[5] M. Green and R. Lazarsfeld, Some results on the syzygies of finite sets and algebraic curves, Compositio Math., 67 (1988), 301-314.

[6] R. Lazarsfeld, A sampling of vector bundles techniques in the study of linear series, in: Lectures on Riemann Surfaces, Proceedings of the College on Riemann Surfaces, International Center for Theoretical Physics, Trieste, Italy, 9 Nov. - 18 Dec. 1987, pp. 500-559, World Scientific (1989). 
[7] F.-O. Schreyer, Green's conjecture for general p-gonal curves of large genus, in: Algebraic Curves and Projective Geometry, Proceedings, Trento 1988, pp. 254-260, Lect. Notes in Math., 1389 Springer-Verlag, Berlin (1989).

[8] E. Sernesi, On the existence of certain families of curves, Invent. Math., 75 (1984), 25-57.

Received March 17, 1993.

UNIVERSITY OF TRENTO

ITALY 

Rosa M. Miró-Roig, Singular moduli spaces of stable vector bundles on $\mathbf{P}^{3}$

Hitoshi Moriyoshi and Toshikazu Natsume, The Godbillon-Vey Cyclic Cocycle and Longitudinal Dirac Operators

J.C. Naranjo, The positive dimensional fibres of the Prym map

Artur Nicolau and Arne Stray, Nevanlinna's coefficients and Douglas al-

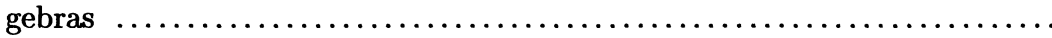

K.K. Park, Entropy of a skew product with a $Z^{2}$-action $\ldots \ldots \ldots \ldots \ldots .227$

María Cristina Pereyra, Sobolev spaces on Lipschitz curves ......... 553

T. Sano, Commuting co-commuting squares and finite dimensional Kac algebras

H.B. Thompson, Second order ordinary differential equations with fully nonlinear two point boundary conditions

H.B. Thompson, Second order ordinary differential equations with fully nonlinear two point boundary conditions II

F. $\mathrm{Xu}$, The flat part of non-flat orbifolds

Hidenobu Yoshida, A type of uniqueness for the Dirichlet problem on a half-space with continuous data 


\section{PACIFIC JOURNAL OF MATHEMATICS}

Volume $172 \quad$ No. $2 \quad$ February 1996

On the failure cycles for the quadratic normality of a projective variety

307

EdoARdo BaLlico

On the minimal free resolution of general embeddings of curves

315

EdOARDO BALlico

On normality of the closure of a generic torus orbit in $G / P$

321

ROMUALD DABROWSKI

Paragroupe d'Adrian Ocneanu et algèbre de Kac

331

MARIE-Claude DAVID

Irreducibility and dimension theorems for families of height 3 Gorenstein algebras

SuSAN J. DiESEL

On the cohomology of the Lie algebra $L_{2}$

ALICE FIALOWSKI

Generic differentiability of convex functions on the dual of a Banach space

John R. Giles, P. S. Kenderov, WarRen Brian Moors and S. D.

SCIFFER

Moon hypersurfaces and some related existence results of capillary hypersurfaces

without gravity and of rotational symmetry

FEI-TSEN LIANG

Stable relations. II. Corona semiprojectivity and dimension-drop $C^{*}$-algebras

TERRY ATHERTON LORING

Singular moduli spaces of stable vector bundles on $\mathbf{P}^{3}$

Rosa M. MIRó-ROIG

The Godbillon-Vey cyclic cocycle and longitudinal Dirac operators

Hitoshi MoRIYOSHI and TOSHIKAZU NATSUME

Nevanlinna's coefficients and Douglas algebras

ARTUR NiCOLAU and ARNE STRAY

Sobolev spaces on Lipschitz curves

MARÍA CRISTINA PEREYRA

A type of uniqueness for the Dirichlet problem on a half-space with continuous data 